\title{
RETOS PARA LA DOCENCIA EN MEDICINA AEROESPACIAL EN COLOMBIA
}

\author{
LINA M SÁNCHEZ RUBIO \\ Médico y Cirujano, Universidad Militar Nueva Granada. Especialista en Otorrinolaringología, \\ Universidad Militar Nueva Granada. Especialista en Docencia Universitaria, \\ Universidad Militar Nueva Granada. Doctora en Salud Pública, Universidad Nacional de Colombia. \\ Directora Centro de Medicina Aeroespacial, \\ Fuerza Aérea Colombiana. \\ Correspondencia: linaorl@yahoo.com
}

Recibido: Enero 7 de $2014 \quad$ Aceptado: Abril 23 de 2014

\begin{abstract}
Resumen
La medicina aeroespacial es una especialidad médica de reciente creación de la cual existen pocos programas en el mundo, uno de ellos en Colombia. La incorporación de los egresados de este programa de posgrado al campo laboral aeronáutico no ha sido un proceso fácil, lo cual lleva a revisar si desde la universidad se están generando los profesionales que la sociedad necesita para un campo altamente especializado como el sector aeronáutico. Desde este ensayo se plantea la propuesta de la formación de un profesional integral para la medicina aeroespacial en Colombia y se formulan algunas estrategias que se pueden implementar desde la educación formal en docencia universitaria.
\end{abstract}

Palabras clave: Docencia universitaria, medicina aeroespacial, formación integral

\section{CHALLENGES IN COLOMBIAN AEROSPACE MEDICINE TEACHING}

\begin{abstract}
Aerospace medicine is a medical specialty with few programs in the world, one of these carried out in Colombia. The incorporation of these graduates to the aeronautical field has not been an easy process, which leads to review whether the university is producing professionals that society needs for a field as highly specialized as aeronautical industry. This paper presents the proposal of a professional with comprehensive training for aerospace medicine in Colombia and proposes some strategies that can be implemented from formal education in university teaching.

Keywords: Aerospace medicine, comprehensive training, university teaching.
\end{abstract}




\title{
DESAFIOS PARA O ENSINO NA MEDICINA AEROESPACIAL NA COLÔMBIA
}

\begin{abstract}
Resumo
A medicina aeroespacial é uma especialidade médica de recente criação, da qual existem poucos programas no mundo; um destes na Colômbia. A incorporação dos professionais ao campo laboral aeronáutico não tem sido um processo fácil, o qual leva a pesquisar se desde a universidade se estão gerando os professionais que a sociedade precisa em uma área altamente especializada como o setor aeronáutico. $\mathrm{O}$ artigo discute a proposta da formação de um profissional integral para a medicina aeroespacial na Colômbia, e sugere algumas estratégias para a implementação desde a educação formal em docência universitária.
\end{abstract}

Palavras-chave: Docência universitária, medicina aeroespacial, formação integral

\section{Introducción}

La medicina aeroespacial es una especialidad médica que surge como requerimiento del desarrollo de la industria aeronáutica y espacial, con el fin de generar las condiciones óptimas para el desempeño humano en estos ambientes especiales. Es por tanto una especialidad de reciente creación, para muchos desconocida, de la cual existen pocos programas en el mundo, uno de ellos en Colombia $(1,2,3)$.

La medicina aeroespacial en el país se enseña como programa de posgrado en la modalidad de curso o diplomado desde hace aproximadamente 25 años, y en el año 2003 se dio comienzo a un programa de especialización médica, de características únicas en Latinoamérica, el cual tiene una duración de tres años, depende del área de medicina interna y comprende rotaciones por ciencias básicas, diferentes especialidades médico-quirúrgicas, áreas afines como medicina preventiva, salud ocupacional y salud pública, y un componente teórico-práctico en aviación (4).

Desde su inicio este programa de especialización generó grandes expectativas nacionales, al tener una concepción interinstitucional y multidisciplinaria, que incluye rotaciones por entidades de aviación civil y de aviación militar en Colombia y una pasantía internacional que permite interactuar con grupos de excelencia en investigación y profundizar en los contenidos académicos de uno de los programas de mayor reconocimiento en medicina aeroespacial de los Estados Unidos. Sin embargo, la incorporación de estos nuevos especialistas al medio laboral aeronáutico en Colombia, el cual es un campo reducido, altamente especializado y competitivo, no ha sido un pro- ceso fácil. En consecuencia, el desarrollo de un programa de especialización como el de medicina aeroespacial en el país plantea importantes desafíos que llevan a reflexionar acerca de la importancia del mejoramiento continuo de los procesos educativos y de la educación formal en docencia universitaria, que para este caso en particular, contribuya a promover profesionales que generen un aporte estructurado a las comunidades científica y aeronáutica a nivel nacional e internacional.

Valorar el aprendizaje de un estudiante es sin duda uno de los aspectos más difíciles de la práctica docente $(5,6,7)$, si se entiende la responsabilidad que representa el entregar a la sociedad los profesionales que necesita, quienes además de contar con suficientes conocimientos y habilidades, deben saber cómo emplearlos para el beneficio de una población determinada, así como en diferentes contextos, lo que obliga a revisar cada una de las fases del proceso enseñanza-aprendizaje $(8,9,10,11,12)$.

Por tanto, en este artículo se plantea que la formación de un profesional integral se constituye en uno de los principales retos para la educación médica $(13,14,15,16)$, y de manera particular para la docencia en medicina aeroespacial en Colombia. Es decir, la formación de un especialista que incorpore tanto el conocimiento generado por la investigación científica desde diferentes disciplinas, como aquel que se obtiene para el caso en mención del saber propio de la cultura aeronáutica. Este último proporcionado esencialmente por el modo de vida y las experiencias de los diversos actores sociales que hacen parte de la aviación, lo cual puede contribuir a optimizar el desempeño en contexto $(17,18,19,20)$. Esto es, un profesional con capa- 
cidad tanto de transmitir como de generar conocimiento, pero esencialmente de llevar ese conocimiento a una práctica efectiva, por lo cual se exponen algunas estrategias de intervención que desde la educación formal en docencia universitaria podrían ayudar para alcanzar estos fines.

\section{Adopción de un modelo pedagógico a través de una estrategia de complementación}

Los procesos educativos constituyen una consideración importante dentro de los retos y oportunidades que se plantean a la medicina aeroespacial para el milenio $(21,22,23,24)$. Cuando se decide asumir el compromiso de la docencia siempre hay que preguntarse qué se quiere que los estudiantes aprendan, cuáles serán los propósitos y fines de este aprendizaje y cómo se podrían alcanzar, lo que lleva a enmarcarse dentro de referentes conceptuales y metodológicos a través de los cuales se va a orientar un programa educativo, es decir establecer el modelo pedagógico que guiará el proceso enseñanza-aprendizaje.

El estudiar en profundidad los diferentes modelos pedagógicos, sus fundamentos teóricos y estrategias didácticas, puede generar aportes sustanciales a la práctica docente en medicina aeroespacial $e$ impactar favorablemente la educación acorde a los requerimientos actuales de la aviación. Es por eso conducente resaltar la importancia de la preparación académica formal para la docencia, que permita identificar los aportes y complementariedad de las diferentes perspectivas desarrolladas a través de la historia para considerar la adopción de un modelo integrado $e$ integral que contribuya a mejorar el desempeño del profesorado, optimice el aprendizaje de los nuevos especialistas y los conduzca a una práctica profesional adecuada a los requerimientos en salud para las operaciones aéreas.

Al intentar la identificación de los aspectos que puedan conducir a ser mejores docentes, se aprecia que no existe un enfoque o aproximación que logre abarcar todo lo que en la práctica se considera necesario. Como lo plantean estudiosos de la pedagogía y personas de gran experiencia en el área educativa $(25,26,27,28,29)$, en el estudio de las diferentes propuestas de modelos pedagógicos se identifican tanto fortalezas como debilidades, y en todos ellos se podrían resaltar aspectos útiles para el proceso de enseñanza-aprendizaje que vale la pena considerar para enriquecer el desempeño docente, y por tanto disciplinar en medicina aeroespacial.

En primer lugar están los aportes desde la escuela tradicional $(25,26)$, llamada también de aprendizaje en tercera persona o modelo pedagógico hetero-estructurante, caracterizada por metodologías receptivas como las clases magistrales, la cual muestra un énfasis particular en aspectos conductuales, autoridad y disciplina. Aunque esta escuela constituye aún un modelo preponderante para la educación en ciencias básicas de la mayoría de facultades de medicina, es blanco de fuertes críticas por considerarse simple transmisión de información centrada en el profesor y la enseñanza, lo que le da un carácter pasivo al estudiante, memorístico, limitante del desarrollo del pensamiento, la autonomía y la creatividad $(28,29)$. No obstante, gran parte de los médicos en el país han sido educados desde esta aproximación, muchos de ellos con un desempeño considerado efectivo dentro de su área de trabajo, y es por tanto también el modelo más empleado en su actividad docente, principalmente si no se ha recibido educación formal en docencia universitaria.

Sin embargo, desde la escuela tradicional se pueden identificar algunas fortalezas para la educación en medicina, $y$ de manera particular en medicina aeroespacial, que no pueden ser pasadas por alto. El médico aeroespacial debe poseer bases conceptuales sólidas de su especialidad, y acceder al conocimiento adquirido y validado a través de diversos años de investigación científica médica y aeronáutica, fundamental cuando se trabaja en áreas como la certificación de aptitud psicofísica de tripulaciones, controladores de tránsito aéreo y personal a cargo del mantenimiento técnico de las aeronaves, como también en el entrenamiento fisiológico para el vuelo, la evaluación de pasajeros y pacientes que requieren ser transportados por vía aérea, las consideraciones de sanidad aeroportuaria, la investigación de accidentes, y el estudio de los factores humanos para la seguridad operacional, que diariamente desafían el conocimiento y criterio médicos. Así mismo, la política de armonización en aviación que busca reducir la accidentalidad por error humano, se orienta esencialmente a la disciplina, el cumplimiento de normas y regulaciones, y a la estandarización de procedimientos que fomenten una cultura de seguridad $(30,31,32,33,34)$.

Por tanto, un aspecto esencial para este tipo de enfoques es como se asume el papel de profesor, el cual debe ser un especialista no solo del área que enseña sino de la docencia $(35,36)$. No es implícito que la escuela tradicional tenga que ser pasiva y memorística, ya que una metodología receptiva donde se privilegian las clases magistrales no es equivalente a los aprendizajes carentes de calidad o significancia (37). Esto permite resaltar que la efectividad de una clase magistral depende mucho de la dinámica impartida por el docente y de las estrategias didácticas 
empleadas. Es posible también a través de estas metodologías compartir con los estudiantes casos reales, en textos o videos, y reportes de investigación, como por ejemplo las experiencias de salud y factores humanos en aviación, que puedan colocarlos en contexto, favorecer la comprensión e impartir más dinamismo a las clases magistrales. Además, en busca de un enriquecimiento mutuo, hay por supuesto que escuchar también las experiencias $e$ intereses de los estudiantes, que constituyen una parte fundamental del constante aprendizaje de un docente para el perfeccionamiento de las actividades académicas. Los intereses de los médicos que aspiran a especializarse en el campo de la medicina aeroespacial son diversos, y van desde la asistencia, la educación y la investigación, hasta la participación en las operaciones aéreas.

Es entendido que dentro de los principales factores para el éxito de un educador están el conocimiento óptimo del área que enseña y la capacidad de despertar el interés de los estudiantes $(11,36,37)$. La claridad conceptual, experiencia disciplinar y apertura al diálogo del personal docente son reconocidos como aspectos clave para multiplicar el conocimiento. En consecuencia, un buen maestro requiere vocación, dedicación, responsabilidad y amor por su trabajo para establecer una dinámica que supere la simple transmisión de conocimientos, presentando conceptos en una forma lógica y ordenada e indicando además las principales fuentes de acceso a dicho conocimiento. Se deben generar por supuesto espacios que permitan el debate, induzcan al pensamiento crítico, la reflexión y el análisis, tanto desde los planteamientos expuestos en las aulas, como desde una juiciosa revisión bibliográfica que incluya lecturas recomendadas por el profesor o sugeridas por el propio estudiante. Por consiguiente, contrario a lo que puedan plantear opositores de la escuela tradicional, vale la pena considerar que un alumno si puede aprender de su profesor, como también un profesor aprende permanentemente de sus estudiantes, para rescatar muchos de los importantes aportes que ha dejado este enfoque pedagógico.

Sin embargo, cuando se trata de un campo como la salud en aviación, que requiere desenvolverse entre fronteras disciplinares, los modelos tradicionales muestran también importantes desafíos que llevan al estudio de nuevas propuestas que enriquezcan la formación académica. Tanto en la docencia como en el ejercicio de la medicina aeroespacial se necesita la interacción constante con personas que pertenecen a diferentes campos del saber en salud y operaciones aéreas, por lo cual es pertinente enfatizar en otro de los factores que llevan a ser un buen maestro, la comprensión de los conocimientos previos del alumno $(36,37)$. Como ya se planteó, al igual que en aviación, en medicina aeroespacial hay que tener claridad conceptual sobre aspectos muy trabajados por el personal de vuelo, como disciplina (por convicción, no por restricción), comportamiento seguro y procedimientos estandarizados, así como el énfasis constante en la comunicación asertiva, el trabajo en equipo y la gestión de riesgos. Por esta razón, para impactar favorablemente todos estos es esencial el concurso articulado de los profesionales en medicina aeroespacial, operaciones de vuelo, educación aeronáutica y seguridad operacional. Esto exige la constante interacción entre áreas del conocimiento, la cual debe trabajarse en principio desde las aulas, para pasar con posterioridad a estrategias más activas que prioricen el concepto integral de salud (38).

Esto hace relevante considerar como en pedagogía cada vez toman más fuerza las escuelas de tipo participativo, los enfoques centrados en el alumno y su desempeño, también conocidos como modelos de aprendizaje en primera persona o auto-estructurantes, dentro de los cuales están la escuela activa y los modelos constructivistas, que hacen especial énfasis en aspectos cognitivos y en el aprendizaje por descubrimiento $(25,26,28,35)$. Estas propuestas tienden a ser más integrales, abarcan diferentes dimensiones y han impulsado enfoques como el aprendizaje basado en problemas y los modelos sociohistóricos $(39,40)$.

Se identifican como principales fortalezas desde estas propuestas el interés por desarrollar el potencial del estudiante, estimular la capacidad de innovación y la generación de nuevo conocimiento. El docente cambia de función, no es el eje sino el acompañante del proceso, se considera como un mediador en el encuentro del alumno con el conocimiento, con el fin de formar personas autónomas y críticas, capaces de transformar la realidad, con habilidades para la comunicación y el trabajo en equipo $(9,39,41)$.

Sin embargo, también estos modelos deben usarse con precaución, pues su adopción no garantiza la calidad en el aprendizaje, ya que se sabe que este depende principalmente de la motivación e interés del estudiante, cuya capacidad no puede ser sobrestimada $(11,37)$. Entonces, se resalta nuevamente la importancia particular del papel desempeñado por el docente, quien debe contar con el conocimiento y el tiempo apropiados para aplicar asertivamente este tipo de aproximaciones y efectuar una adecuada asesoría que facilite el desarrollo de criterio y autonomía. Además, es oportuno señalar que la adopción de propuestas educativas que exigen flexibilización, no implica que se deje de establecer orden y coherencia 
dentro de un programa académico, sino que el estudiante comprenda y se motive para hacer parte fundamental en la construcción del conocimiento.

La comprensión del entorno en el cual se desenvuelven las operaciones aéreas es fundamental para entender los problemas de salud en aviación. Esto se fortalece al impulsar el mejoramiento continuo de las estrategias didácticas que favorezcan el aprendizaje basado en problemas y la contextualización de la práctica médica. Por ejemplo, en una escuela integrada de medicina aeroespacial y operaciones médicas especiales es importante emplear escenarios de simulación, para recrear situaciones y condiciones fisiológicas como las de la vida real en aviación (24), así como el aprendizaje continuo en el trabajo diario con pacientes a través de una práctica coherente. En el país se identifican diferentes escenarios que pueden enriquecer el trabajo práctico de quienes se encuentran en proceso de especialización en medicina aeroespacial, que permiten interactuar directamente con la problemática a intervenir, como son las escuelas de formación aeronáutica, los centros de atención médica, las áreas de operación de vuelo, las diferentes dependencias de la autoridad aeronáutica, las empresas y la industria. Es decir, como lo sugieren expertos que han profundizado en el análisis de los retos para la educación $(27,35,42)$, hay que propender por el desarrollo de pedagogías para la comprensión desde potencialidades, ambientes y estudiantes específicos.

En este punto es propicio mencionar los valiosos aportes desde la educación por competencias, implementada con gran aceptación en las instituciones de educación superior, que mira diferentes dimensiones del saber y permite una actividad más efectiva al estructurar un plan de trabajo en un programa académico. Se puede decir que el enfoque de educación por competencias imparte dinamismo a la práctica docente, coherente con la complejidad que caracteriza a los seres humanos $(43,44,45)$.

Se aprecia por tanto que las diferentes propuestas que buscan mejorar los procesos educativos no son excluyentes sino complementarias. Además, no siempre un modelo determinado se ajusta a todos los estudiantes, ni tampoco a todos los docentes, lo que conduce a intentar identificar o construir el que se adapte mejor a las necesidades y capacidades, procurando que sea práctico y efectivo. Para el caso específico de la medicina aeroespacial, proyectar especialistas integrales que trabajen por el bienestar y calidad de vida de las tripulaciones de vuelo con el fin de contribuir a su adecuado desempeño, profesionales que incorporen la salud a la seguridad operacional aérea enfrentando los desafíos humanos que plantea la industria de aviación, a través de la comprensión de los diferentes contextos operacionales y niveles del sistema aeronáutico. Dentro de los diversos retos a enfrentar se pueden mencionar algunos como la adaptación a un medio ambiente extremo, la definición de criterios de aptitud psicofísica, la estandarización en un entorno multicultural, la automatización de las cabinas, las aeronaves remotamente tripuladas, las operaciones permanentes, los vuelos espaciales, el manejo del estrés y la fatiga.

Por tanto, debido a la complementariedad identificada si se dedica tiempo a estudiar las diferentes perspectivas en educación, vale la pena considerar la aproximación a un modelo integrado e integral, con aportes tanto de la escuela tradicional como de los enfoques esencialmente participativos, de las pedagogías clásicas y las pedagogías críticas, como lo señalan algunas propuestas pedagógicas $(9,26,27,28,35,40,42,46)$, que despierten el interés y desarrollen la inteligencia con el fin último de alcanzar el mejor beneficio para los estudiantes como constructores del presente y del futuro, como personas con responsabilidad social, preocupadas por la solución de los problemas que enfrenta la salud, considerando aspectos sociales, políticos, económicos y éticos, la cultura y prácticas de los profesionales de la salud y de quienes son impactados por su trabajo, en el caso de la medicina aeroespacial, el personal de vuelo. Esto exige un ejercicio transdisciplinar entre las ciencias médicas, sociales y humanas (47), que lleve con la participación de docentes y estudiantes a construir un modelo pedagógico congruente y coherente con una adecuada práctica médica, estableciendo una dinámica que entendiendo el contexto operacional contribuya a identificar de manera permanente acciones que lleven al mejoramiento continuo de la calidad en salud y en las operaciones de vuelo.

\section{Enseñanza y aprendizaje hacia la responsabilidad social y la ética operacional}

Dentro de la propuesta de integralidad que debe caracterizar a un especialista en medicina aeroespacial un aspecto esencial para cualquier profesional de la salud es su formación humanista(14,42,48,49), entendiendo que esta debe impactar de manera transversal cualquier modelo que se adopte para la formación de estos especialistas, priorizando en este caso la ética en salud y seguridad operacional, impulsando una relación médico-paciente soportada en el respeto, la confianza y la confidencialidad, en el marco de una cultura de seguridad permeada por principios y valores. 
Como se señaló, no cabe duda que un buen médico debe contar con sólidos conocimientos científicos sobre su campo del saber, así como conocer del empleo adecuado de todos los recursos tecnológicos y terapéuticos, quirúrgicos o farmacológicos al servicio de la medicina, entendidos bajo nuevas propuestas en educación como competencias del saber y del hacer, por tanto, esta es el área que ha ocupado la mayor parte del currículo en esta profesión. Pero al asumir el reto de una formación integral, además de la adquisición y empleo de conocimientos, la educación debe promover el desarrollo de las potencialidades humanas, como individuos y como seres sociales. Es por eso que los egresados deben desarrollar también competencias del ser, el hacer saber y el saber convivir, obteniendo finalmente una división funcional en procesos de aprendizaje, valores sociales, contexto tecnológico e internacional y habilidades interpersonales $(43,44)$.

Entonces, hay que trabajar por llevar a la práctica este desarrollo teórico en la educación. De acuerdo a esto, es punto de reflexión como el ejercicio de la medicina en esencia suele desarrollarse alrededor de la enfermedad, conduciendo al abandono de la integralidad que conlleva el concepto salud, que está más orientado a la generación de bienestar y calidad de vida $(50,51,52)$, privilegia al humano como individuo y como ser que vive en colectividad, y llevaría en este caso de práctica en medicina aeroespacial, a considerar diferentes escenarios más allá del consultorio, llevando a una mayor intervención en los problemas sociales que afectan la salud y el desempeño del personal de vuelo en el contexto operacional colombiano, incrementando su campo de acción y los aportes al sector aeronáutico.

Por consiguiente, la educación en medicina debe evitar la inducción al pensamiento reduccionista $(10,12,41,46,53)$, y aunque parezca antagónico más aún si se está en proceso de especialización, para orientar un poco más hacia el pensamiento sistémico con su complejidad inherente, que permita especializarse en las partes sin desconocer el todo, es decir, aprender a articular la generación de conocimiento con la problemática local, regional y global, a través de una estrategia que articule disciplinas y culturas, que se alcanza mejor cuando se aprende a trabajar y solucionar problemas como parte de un equipo.

Se enfatiza por tanto la necesidad de contextualizar la práctica médica para impactar favorablemente los problemas que se plantean a la medicina aeroespacial. Al profundizar en el estudio de las enfermedades y los comportamientos que afectan la salud de los individuos y las poblaciones se puede identificar que estos generalmente están inmersos en aspectos socioculturales de diferente índole, para algunos autores definidos como determinantes sociales de la salud, que deben también ser controlados si se quieren generar verdaderos beneficios $(50,54,55)$. Esto requiere además de conocimientos clínicos y terapéuticos validados, de la comprensión que lleve a una práctica fundamentada en la promoción de la salud y calidad de vida para la prevención de la enfermedad y el acertado desempeño operacional, que oriente a la solución de problemas estructurales, dinámica que debe ser entendida desde el proceso de enseñanza-aprendizaje, conduciendo más hacia el pensamiento crítico que privilegie aquellos enfoques que consideran la salud como valor y como derecho, lo cual debe ser principio fundamental de la educación médica, necesita el compromiso de académicos y estudiantes y la participación de la población a intervenir.

Por lo regular para los especialistas en medicina los abordajes a los problemas en salud basados en evidencia, generalmente muy concretos y técnicos son estimados más prácticos, en cambio los abordajes humanistas y sociales se identifican muy teóricos y etéreos. Pero es conducente en este enfoque resaltar que la salud tiene un componente individual y un componente colectivo, integra lo biológico y lo social, y se manifiesta para el caso de la aviación, desde aspectos fisiológicos, mentales y comportamentales. Adoptando una postura crítica hacia el enfoque dado al manejo de la salud, se puede elucidar el principal énfasis hacia la asistencia, lo individual, lo técnico y la medicalización, que aunque esencial para el ejercicio de la medicina, es solo una de sus partes y se aleja muchas veces de la identificación de la problemática general $(51,54,55)$. Podría decirse entonces que se está fallando en la responsabilidad social que debe tener todo profesional de la salud, y desde los planteamientos expuestos, sería conveniente que apartados de posturas radicales o relativistas se tome en consideración la opción por la complementariedad de perspectivas para generar aportes desde todas las áreas que impactan la salud operacional, en las dimensiones individual, particular $y$ general, para mejorar los procesos de certificación, educación, instrucción y entrenamiento, operaciones aéreas, normas y procedimientos entre otros, todos estos en los cuales tiene injerencia el especialista en medicina aeroespacial.

Esto señala nuevamente la pertinencia que desde la academia se fomente el trabajo en equipo y se generen espacios de diálogo $(10,11,12,36,39,41,46)$ para analizar la problemática de salud en aviación, asociada muchas veces a su rápido desarrollo tecnológico y las demandas de la industria aeronáutica, que permita llegar a través de sanos debates y decisiones consensuadas a la identificación de acciones de mejora. Al respecto, vale la pena analizar 
también la propuesta de incluir áreas como la bioética en el currículo universitario en medicina $(48,56)$. Por ejemplo, uno de los retos más grandes que enfrentan los especialistas en medicina aeroespacial es la toma de decisiones en el proceso de certificación de la aptitud psicofísica para actividades de vuelo, que implica contar no solo con amplios conocimientos clínicos, de fisiología del vuelo y los conceptos fundamentales en aeronáutica y seguridad aérea, sino también interactuar con los diferentes integrantes de la aviación, para mediar en beneficio de la salud y calidad de vida del personal de vuelo y prevenir accidentes. También otro campo donde existen este tipo de interacciones, que demanda una amplia competencia en resolución de problemas y conflictos es la toma de decisiones para el traslado de pacientes por vía aérea, en donde intervienen diferentes profesionales de la salud, del área administrativa y del sector aeronáutico. Además, la aviación se mueve en una dinámica de mercado altamente competitivo y muchas veces los médicos se enfrentan a dilemas éticos en su proceso de incorporación y adaptación al sistema, los cuales se resuelven mejor con el conocimiento del medio y una adecuada preparación académica para poder privilegiar la salud en apoyo al desempeño humano en las operaciones de vuelo.

Hablaríamos entonces de una educación en valores, con el fin de impactar la crisis que afronta en ocasiones la sociedad, donde priman muchas veces las relaciones de poder, y la calidad de vida se sustenta más en el tener que en el ser, para que aspectos como responsabilidad, solidaridad, justicia social, equidad, respeto, tolerancia, confianza y confidencialidad hagan siempre parte de un comportamiento ético coherente con la misión del acto médico.

Es sorprendente identificar como en un mundo globalizado, liderado por las tecnologías de información y las comunicaciones, paradójicamente las personas han perdido su capacidad para relacionarse. Se considera que la supra-especialización de las ciencias genera fragmentación $(13,42,40,55)$, y un aspecto a tener en cuenta como contribuyente de la fragmentación de disciplinas son las fallas en la comunicación, que afecta de manera contundente las relaciones sociales. Este problema de comunicación es de origen multifactorial, pero es evidente que los enfoques cada vez más orientados a lo técnico y procedimental, tanto en las especialidades médicas como en las operaciones aéreas, a pesar de haber generado importantes aportes a la efectividad y seguridad en salud y aviación, si no son empleados con precaución pueden inducir a perder lo esencialmente humano de las relaciones interpersonales. Por ejemplo, tanto en la relación médico-paciente como entre colegas se identifica muchas veces falta de diálogo e incapacidad para establecer una comunicación asertiva. Estas relaciones se encuentran también limitadas en tiempo efectivo, fundamental para una buena comprensión de los problemas de salud y la generación de un apoyo integral a los pacientes. Por eso el fortalecimiento de las relaciones humanas debe ser una estrategia fundamental en la educación universitaria, además del trabajo previo desde los colegios y las familias.

Para el caso en mención, identificar cual es el fin de la práctica en medicina aeroespacial puede ayudar a orientar mejor los objetivos educativos. Al reflexionar sobre el trabajo en salud, con seres humanos y su bien más preciado la vida, se entiende mejor la profesión médica como vocación de servicio. Se trata entonces de retomar la misión humanista de la formación profesional y de atreverse a hacer propuestas transformadoras. Si la universidad trabaja para educar en la comprensión, de acuerdo a los retos planteados para la educación del futuro, se facilitará el camino $(27,35,42)$. Porque somos seres sociales debemos orientar la educación más hacia la colaboración que hacia la competencia, fomentando el trabajo en equipo que lleve a mejores resultados en la toma de decisiones, mediando acuerdos y desacuerdos.

\section{Promoción de docentes investigadores}

Al considerar los fundamentos teóricos que sustentan la práctica docente se entiende mejor la responsabilidad que representa el ser educadores, y por consiguiente la importancia de una adecuada preparación profesional para la docencia en las especialidades médicas, la cual debería incluir en lo posible la formación como investigadores.

Tratándose de un campo profesional tan específico, la mayoría de los profesionales que trabajan en medicina aeroespacial y seguridad aérea en el país están llamados a participar en la educación del personal de la salud y de otras áreas del sector aeronáutico, así como a la solución de su problemática. Sin embargo, al igual que ha sucedido en otras áreas de formación, gran parte de los docentes en esta especialidad ejercen principalmente por vocación y no tienen formación en pedagogía y docencia universitaria, desconociendo los aportes que estas pueden brindar para un mejor desempeño.

Además, se identifican diferentes posturas en cuanto a si se deben integrar o no dos importantes misiones de la universidad, la docencia y la investigación $(13,46,57)$. Quienes están en contra, argumentan que esta integración requiere 
de mayores recursos y tiempo, que es difícil articular estos dos campos en un mismo profesional, se podría afectar la calidad de la docencia y requiere de gran motivación. Sin embargo, quienes están a favor consideran que la docencia y la investigación son dimensiones de un mismo proceso que se potencian, y que el estudiante al descubrir aprende, maximizando la producción académica y científica para lograr los objetivos académicos y sociales $(58,59)$.

Pero al entender las características del campo de la medicina aeroespacial, se exalta la trascendencia de esta integración docente-investigador para una mejor formación de especialistas. Porque la promoción de docentes investigadores que orienten y dirijan líneas y grupos de investigación desde la universidad puede enriquecer de manera fundamental el desarrollo de esta especialidad médica en Colombia como aporte a las comunidades científica y aeronáutica a nivel nacional e internacional, ya que uno de los más importantes desafíos que enfrenta la aviación es el impulso de la investigación científica y la innovación con el fin de generar las condiciones para que los seres humanos interactúen adecuadamente con la tecnología y se desempeñen de manera efectiva en este ambiente especial manteniendo salud y calidad de vida. El humano siempre estará al mando y control de las aeronaves, incluso de las remotamente tripuladas, por eso sus capacidades y limitaciones son motivo constante de investigación, la cual debe ir paralela al desarrollo tecnológico de la industria aeronáutica.

Por tanto, el trabajo en investigación debe ser una competencia esencial de docentes y especialistas en medicina aeroespacial, investigación que debe orientarse tanto al beneficio del área disciplinar como de los procesos educativos. Por ejemplo, en el contexto nacional el incremento de las operaciones aéreas con la incursión de varias empresas multinacionales, la incorporación de nueva tecnología aeronáutica que conlleva a transferencia cultural, así como la amplia experiencia adquirida en operaciones aéreas militares en apoyo a la seguridad nacional, obliga a profundizar en el estudio de la problemática en salud y seguridad de las tripulaciones de vuelo y del impacto de los factores humanos en las operaciones aéreas, así como también en todos los aspectos de salud relacionados con el transporte aéreo de pasajeros y pacientes, para generar conocimiento útil a la solución de diferentes problemas, lo cual debe ser enfatizado desde la universidad y comprendido por estudiantes y docentes como una responsabilidad social.

Es conducente también poner en consideración para los docentes investigadores la importancia de inducir a la transdisciplinariedad en el abordaje de los problemas relacionados a la salud y el estudio de los factores huma- nos en aviación $(28,30,44,45,47,60)$, ya que estos son fenómenos donde confluyen diferentes campos del saber, las ciencias naturales, humanas y sociales, aspectos biológicos, económicos, culturales, etc., y es solo a través de la comprensión integral de esta complejidad que se podrán generar estrategias para impactar aspectos estructurales.

En esta articulación de disciplinas surge también el impulso de investigaciones multimétodo, que integran técnicas cuantitativas y cualitativas, complementando los objetivos y métodos de las diferentes ciencias $(47,60,61)$, que permita orientar mejor las estrategias para la solución de problemas.

Finalmente, el ser docentes investigadores ayudaría a trabajar mejor en la educación de profesionales resilientes, capaces de adaptarse a situaciones cambiantes, induciendo a la flexibilidad cognitiva que les permita contribuir a la generación de conocimiento más allá de lo que muestran los textos, para estar en producción constante de ideas y siempre abiertos a nuevas interpretaciones de la realidad.

\section{Conclusión}

Considerando las reflexiones expuestas se puede concluir que para optimizar la formación de los especialistas en medicina aeroespacial en Colombia se requiere de educadores especializados no solo en las diferentes disciplinas relacionadas sino también en la docencia universitaria, lo que permitiría fortalecer la academia con las herramientas aportadas desde los desarrollos teóricos en educación a través de diferentes perspectivas, las cuales se presentaron en estos planteamientos como complementarias con el fin de formar un profesional integral.

Los retos que se presentan a la docencia en medicina aeroespacial exigen el fortalecimiento de la educación con estrategias que induzcan a aprendizajes significativos en diferentes áreas, es decir, la adquisición de conocimiento útil y pertinente para las necesidades del campo donde se desenvuelve el personal aeronáutico.

Además, como el conocimiento está en construcción permanente, estas estrategias deben llevar a constante formulación de preguntas de investigación para la solución de problemas en beneficio de la población a intervenir y de su área de vida y de trabajo. Por eso se exalta la inclusión de docentes investigadores que puedan ayudar a estos especialistas a incorporarse de una manera más adecuada y eficiente a la dinámica mundial en aviación, campo de muy rápido desarrollo científico y tecnológico, entendiendo su entorno sociocultural. 
El impulso de docentes e investigadores debería ser una condición casi inherente para la formación en medicina aeroespacial, ya que todos los aspectos de salud física, mental y de comportamiento que pueden afectar la adaptación del humano al medio ambiente aeronáutico y espacial, así como la identificación de sus limitaciones y la forma de superarlas, hacen esencial la multiplicación y generación del conocimiento en este ampliamente especializado campo del saber para conducir a una práctica profesional efectiva.

Para médicos especialistas que van a trabajar en un mundo liderado por el desarrollo científico y tecnológico y la gran estandarización y regulación aeronáuticas, así como la medicina basada en la evidencia, los protocolos y las guías de práctica médica, es importante entender que estos son solo referentes de un conocimiento adquirido desde la experiencia operacional y la investigación científica. Los retos constantes que presenta la adaptación del hombre al medio ambiente aeroespacial en su interacción con tecnología en constante innovación, orienta hacia la enseñanza reflexiva y la flexibilidad curricular, con propuestas didácticas y escenarios de práctica que ayuden a la comprensión de la problemática y generación de nuevo conocimiento, que enriquezca la formación recibida desde los enfoques tradicionales. Es decir que para optimizar su desempeño, se debe propiciar en los estudiantes un pensamiento no homogenizado ni homogenizante, sino que induzca al desarrollo de criterio enriquecido por la autocrítica y la reflexión, sin olvidar por supuesto el compromiso y la responsabilidad con el fortalecimiento de la seguridad en las operaciones aéreas, un pensamiento con capacidad para la solución de problemas en la armonía y en la divergencia, el cual es posible si se sitúa en la escala valorativa indicada, es una elección ética.

$\mathrm{Al}$ asumir estos retos para la docencia en medicina aeroespacial se puede proponer como estrategia fundamental de intervención la enseñanza y aprendizaje que promueva el trabajo interinstitucional, interdisciplinario, participativo y práctico, como se mostraría desde el trabajo articulado entre la universidad, la autoridad aeronáutica estatal, las instituciones civiles, militares y las empresas que trabajan en aviación en Colombia, reiterando el intento permanente de cooperación entre los profesionales de la salud y las personas que trabajan en operaciones aéreas, educación aeronáutica y seguridad operacional incluyendo todos los niveles del sistema aeronáutico, como son el regulador, el gerencial, el supervisor, el operativo y la industria. Esto ayudará a la adecuada incorporación en el sistema de especialistas competentes con un aporte efectivo para la problemática en salud y factores humanos en aviación fomentando una práctica transformadora en beneficio de la calidad de vida del personal de vuelo, la efectividad y seguridad en las operaciones aéreas, una responsabilidad que puede ser liderada desde la academia.

Conflicto de intereses: No hay conflictos de intereses para señalar por parte de la autora.

Financiación: El trabajo se financió con fondos propios de la autora.

\section{Referencias}

1. Davis JR, Johnson R, Stepanek J, Fogarty JA. Fundamentals of aerospace medicine. Philadelphia: Lippincott Williams \& Wilkins; 2008.

2. Rainford DJ, Gradwell DP. Aviation Medicine. New York: Hodder Arnold Ltd; 2006.

3. DeHart RL. Aerospace Medicine. En: Wallace RB. Public Health \& Preventive Medicine. New York: McGraw Hill; 2007.

4. Universidad Nacional de Colombia. Medicina Aeroespacial. Un avance en Colombia: pionera en Latinoamérica. Disponible en: http://upload.wikimedia.org/wikipedia/commons/e/e3/ MedicinaAeroespacialUnAvanceenColombia.pdf

5. Jamé M. Evaluación del aprendizaje: ¿problema o herramienta?. Revista de Estudios Sociales. 2004; 20:93-98.

6. González M. Evaluación del aprendizaje en la enseñanza universitaria. Revista Pedagogía Universitaria 2000; 5(2): 1-121.

7. Carrizo W. La responsabilidad del docente frente a la evaluación. Pecunia 2009; 9:63-83

8. Cunningham I, Cunningham EL. La sociología de la medicina ocupacional. Revista de Ciencias Sociales (San Juan) 1989; 28(12): $15-41$.

9. Díaz M. Lectura Crítica de la Flexibilidad, Volumen1: La Educación Superior Frente al Reto de la Flexibilidad. Bogotá: Cooperativa Editorial Magisterio; 2007.

10. Medina JL, Jarauta B, Imbernon F. La Enseñanza Reflexiva en la Educación Superior. Cuadernos de Docencia Universitaria 17. Barcelona: Editorial Octaedro; 2010.

11. Moreno T. Didáctica de la educación superior: Nuevos desafíos en el Siglo XXI. Revista Perspectiva Educacional 2011; 50(2): 26-54.

12. Archila PA. La investigación en argumentación y sus implicaciones en la formación inicial de profesores de ciencias. Revista Eureka sobre Enseñanza y Divulgación de las Ciencias 2012; 9(3): 361375.

13. Ortega y Gasset J. Misión de la Universidad. Con indicaciones y notas para los cursos y conferencias de Raúl J. Palma. Buenos Aires; 2001. 
14. Morales JC. Formación integral y profesionalismo médico: una propuesta de trabajo en el aula. Educ.med.2009; 12(2).

15. Fernández B, Morales I, Portal J. Sistema de influencias para la formación integral de los egresados de los Centros de Educación Médica Superior. Educación Médica Superior 2004; 18 (2): 1-1.

16. Ibarra G. Ética y formación de profesional integral. Reencuentro análisis de problemas universitarios 2005; 43: 32-38.

17. Helmreich RL, Merritt AC. Culture at work in aviation and medicine. National, organizational and professional influences. Hampshire, UK: Ashgate; 1998.

18. Batteau AW. Anthropological approaches to culture, aviation, and flight safety. Human Factors and Aerospace Safety 2002; 2(2): 147-171.

19. Batteau AW, Maurino D, Merritt AC, Schutte P. Culture and aviation safety: Four voices in pursuit a common goal. $12^{\text {th }}$ International Symposium on Aviation Psychology 2003;82-87.

20. Li WC, Harris D, Chen A. Eastern minds in western cockpits: meta-analysis of human factors in mishaps from three nations. Aviat Space Environ Med 2001; 78:420-5.

21. Grenon SM, Saary J. Challenges in aerospace medicine education. Aviation, Space and Environmental Medicine 2011;82: 10711072

22. Doarn CR, Mohler SR. Physician training in aerospace medicinean historical review in the United States. Aviation, Space and Environmental Medicine 2013; 84:158-162.

23. Rayman RB. Aerospace Medicine: Challenges and Opportunities. Aviation, Space, and Environmental Medicine 2005; 76: 992-996.

24. Mohler S, Nicogossian A. Training aerospace medicine physicians in the US. Aviation, Space and Enviromental Medicine 1986;57(4): 378-380.

25. Gadotti, M. Historia de las ideas pedagógicas. México D.F.: Siglo XXI editores; 2005.

26. De Zubiría J. Los Modelos Pedagógicos: Hacia una Pedagogía Dialogante. 2ª̊Ed. Bogotá: Editorial Magisterio; 2005.

27. Vasco CE. Pedagogías para la comprensión en las disciplinas académicas. Uni-pluri/versidad. Universidad de Antioquia 2001; 1(3):19-28.

28. Isaza, A. Clases magistrales versus actividades participativas en el pregrado de medicina. De la teoría a la evidencia.Rev.Estud. Soc.2005; No.20.

29. Ruiz, F.J. Modelos didácticos para la enseñanza de las ciencias naturales. Latinoam estud educ Manizalez (Colombia) 2007; $3(2): 41-60$.

30. Wiegmann DA, Zhang H, Von Thaden TL, Sharma G, Gibbons AM. Safety culture: An integrative review. The international Journal of Aviation Psychology 2004; 14(2): 117-134

31. Organización de Aviación Civil Internacional. Manual de gestión de la seguridad operacional. Primera edición, 2006.

32. Bennet SA. A sociology of commercial flight crew. Hampshire, UK: Ashgate; 2006

33. Dekker S.W.A. The re-invention of human error. Lund University School of Aviation.Tech Report 2002-01;1-16.

34. Sánchez LM. Centro de Medicina Aeroespacial: Pilar de la efectividad operacional. Revista Aeronáutica. Fuerza Aérea Colombiana 2011; Edición 263: 6-9.
35. Vasco CE. Siete retos para la educación colombiana para el periodo 2006 a 2019. Conferencia dictada para la universidad EAFIT de Medellín el 10 de marzo de 2006. Disponible en: http://www.eduteka.org/RetosEducativos.php

36. Bain K. Lo que hacen los mejores profesores universitarios. Universidad de Valencia 2007; 26-32.

37. Dávila, S. . El Aprendizaje Significativo: Esa extraña expresión (utilizada por todos y comprendida por pocos). Contexto educativo Julio 2000; No. 9. Disponible en: http://contexto-educativo.com. ar/2000/7/nota-08.htm

38. Sánchez, L.M. Medicina aeroespacial y factores humanos en aviación. La importancia de una aproximación transdisciplinaria a la salud. Revista Med 2008;16(2): 249-260.

39. Servicio de Innovación Educativa de la Universidad Politécnica de Madrid. Aprendizaje Basado en Problemas. Guías rápidas sobre nuevas metodologías. Madrid: UPM 2008; 4-14.

40. Aronowitz S, Giroux H. Postmodern Education: Politics, Culture and Social criticism. Minneapolis: University of Minnesota Press; 1993.

41. Servicio de innovación educativa de la Universidad Politécnica de Madrid. ¿Qué es el Método del Caso?. Madrid: UPM; 2008.

42. Morin E. Los siete saberes necesarios a la educación del futuro. Paris: UNESCO; 1999.

43. Beneitone P, Esquetini C,González J,Maletá MM, Siufi G, Wagenaar R. Reflexiones y perspectivas sobre la educación superior en América Latina. Informe final- Proyecto Tunning-América Latina 2004-2007. Universidad de Deusto-Universidad de Groningen; 2007.

44. Salas WA.Formación por competencias en educación superior Una aproximación conceptual a propósito del caso colombiano. Revista iberoamericana de educación 2005; 36(9): 1-11.

45. Schmal R, Ruiz-Tagle A. Una metodología para el diseño de un currículo orientado a las competencias. Ingeniare. Revista chilena de ingeniería 2008; 16(1): 147-158.

46. Ordoñez F. Pedagogía crítica y educación superior. Revista Educación 2002; 26(2): pp. 185-196.

47. Sánchez LM. Medicina aeroespacial y factores humanos en aviación. La importancia de una aproximación transdisciplinaria a la salud. Revista Med 2008;16(2): 249-260.

48. Outomuro D. Fundamentación de la enseñanza de la bioética en medicina. Acta Bioéthica 2008;14(1): 19-29

49. Berry JW. Psychology of group relations: Cultural and social dimensions. Aviat Space Environ Med 2004; 75 (7,Suppl.): C52-7

50. Buck C. Después de Lalonde: la creación de la salud. En: Organización Panamericana de la Salud-OPS. Promoción de la Salud: una antología. Publicación científica No557. Washington DC: OPS/OMS 1996; 6-14

51. Franco S. La salud pública hoy. Enfoques y dilemas contemporáneos en salud pública. Bogotá: Universidad Nacional de Colombiana; 2003.

52. Wallace RB. Public Health \& Preventive Medicine. New York: McGraw Hill; 2007.

53. Henao BL, Sptipcich MS. Educación en ciencias y argumentación: la perspectiva de Toulmin como posible respuesta a las demandas y desafíos contemporáneos para la enseñanza de las ciencias experimentales. Revista Electrónica de Enseñanza de las Ciencias 2008; 7(1), 47-62. 
54. Marmot M, Richard GW. Social determinants of health. Oxford: Oxford University Press; 1999

55. Breilh J. Epidemiología crítica: Ciencia emancipadora e interculturalidad. Buenos Aires: Lugar Editorial; 2003

56. Villegas EM. Tendencias de la formación bioética en los currículos universitarios. Revista Colombiana de Bioética 2011; 6(1), 103124.

57. Lozano G. La articulación entre investigación y docencia en la Universidad de Antioquia. Revista Educación y Pedagogía 2006; 18(46), 91-99.
58. Del Huerto VM. Docencia e Investigación: Un encuentro necesario y posible. Ponencia presentada en el IV Encuentro Nacional de Docentes Universitarios Católicos-ENDUC; 2007.

59. Gonzalez-Sistal A, Gomez JM. Metodología docente aplicada a la medicina aeroespacial. Visión actual. Medicina Aeroespacial y Ambiental 2004; 4(2), 71-76.

60. Sánchez LM. El factor humano en accidentes de aviación. Revista Pensamiento Psicológico. Universidad Javeriana Cali 2010; 7(14): 141-153.

61. Brewer, J. \& Hunter, A. Foundations of multimethod research. Synthesizing styles. London: Sage Publications Inc.; 2006. 